\title{
Comprehensive Partitions and Optimization Strategies Based on Tourism Urbanization and Resources and Environment Carrying Capacity in the Yellow River Basin, China
}

\author{
Zhaofeng Wang \\ Hunan Normal University \\ Qingqing Chen ( $\nabla$ lightcqq@126.com ) \\ Hunan Normal University https://orcid.org/0000-0003-2571-2648
}

\section{Research Article}

Keywords: Tourism urbanization, resources and environment carrying capacity, partitions, optimization strategies, Yellow River Basin

Posted Date: June 16th, 2021

DOl: https://doi.org/10.21203/rs.3.rs-521525/v1

License: (c) (1) This work is licensed under a Creative Commons Attribution 4.0 International License. Read Full License 


\title{
Comprehensive partitions and optimization strategies based on tourism urbanization and resources and environment carrying capacity in the Yellow River Basin, China
}

\author{
Zhaofeng Wang ${ }^{1} \cdot$ Qingqing Chen ${ }^{1}$ \\ First author: Zhaofeng Wang \\ Corresponding author: Qingqing Chen \\ E-mail: lightcqq@126.com \\ Tel: 15348448627 \\ ${ }^{1}$ College of Tourism, Hunan Normal University, Changsha 410081, Hunan, China
}

\begin{abstract}
A better understanding on the spatial matching relationship between tourism urbanization and resources and environment carrying capacity is vital for the regional selection of the key eco-livable tourist cities in the Yellow River Basin. This paper addressed this research issue by evaluating and partitioning tourism urbanization level and resources and environment carrying capacity of the Yellow River Basin in 2005, 2011 and 2018, using GIS technology, spatial autocorrelation model and partition method. Empirical results suggest that (1) The tourism urbanization level of Shaanxi province maintains the leading position, while Shanxi province has great development potential. The high value areas of resources and environmental carrying capacity concentrated in Gansu, Inner Mongolia and Shandong provinces. (2) The spatial agglomeration degree of tourism urbanization level and resources and environment carrying capacity have been improved. (3) The resources and the environment carrying capacity exhibits a greater restrictive effect on the current high-level areas of tourism urbanization, and the spatial correspondence between them is weak. Based on the findings of this study, a series of optimization strategies and policy suggestions have been proposed for promoting the sustainable development of tourism urbanization in the Yellow River Basin.
\end{abstract}

Keywords Tourism urbanization . resources and environment carrying capacity · partitions · optimization strategies · Yellow River Basin 


\section{Introduction}

In many countries, tourism urbanization is considered as an effective means to prevent agricultural decline and promote the transformation and upgrading of urban industries (Wei 2017). In China, tourism urbanization is a key strategy and one of the main targets for new-type urbanization. It influences and shapes the urbanization development in many aspects (Tao et al. 2017). The ecological protection and high-quality development of the Yellow River Basin have become China's major national initiative in 2019 (Xinhua News Agency, 2019). For major national strategic region with geographical features such as national concentrated contiguous poverty-stricken areas, national ecological function areas and tourism resource-rich areas, the contribution of tourism sector to urbanization construction is more critical. However, there is few in-depth investigations in the relevant literature. Meanwhile, the ecological environment of the Yellow River Basin is relatively fragile, and the carrying capacity of resources and environment is relatively low, which has a strong binding effect on the development of tourism urbanization. Therefore, how to accelerate the development of tourism urbanization in the Yellow River Basin taking into account the effects of resources and environment, and the sustainable development of tourism urbanization has become an urgent problem in the area.

The tourism urbanization and resources and environment carrying capacity are dynamic process of development. They both have promotion and constrain effects against each other. This is reflected in the nonlinear, complex and dynamic characteristics of its behavior (Raza et al. 2021 ). On the one hand, the orderly development of tourism urbanization can provide financial and technical support for the resources and environment system, which is conducive to the rational development of resources and protection of the environment. The disordered development of tourism urbanization, however, will also destroy the ecological resources and overload the ecological environment capacity(Xie et al. 2013). On the other hand, the resources and environment system brings resources supply and environmental support for the development of tourism urbanization, but it also restricts the development scale of tourism urbanization(Izquierdo et al. 2018; Nitivattananon and Srinonil 2019).

The sustainable development of tourism urbanization and ecological protection, and their interactions, has been a hot issue in the academic research and industry practice (Zhang et al. 2020) . With the expansion of international hotels in Fiji, the increasing demand for tourism development and rapid urbanization have led to environmental degradation, extensive land use, and loss of coastal vegetation, which in turn has affected local communities adversely (Xie et al. 2013). Rai \& Goswami (2019) observed that tourism and urbanization have placed enormous pressure on Gantok's infrastructure services and environment, particularly in the area of waste management. González et al. (2014) found an inverse relationship between increases in the level of urbanization and the abundance of beetles. These studies focused on the disruption and destruction of the eco-environment through tourism urbanization activities, as a one-way process. The coordinated relationship between tourism urbanization activities and ecology have not been comprehensively discussed. The relationship between tourism urbanization and ecological environment is a two-way mechanism, and maintaining a regionally-specific balance is essential. 
Meanwhile, researchers have also analyzed the coupling coordination degree of tourism, urbanization and ecological environment. Zhang and $\mathrm{Li}$ (2020) detected that the coupling degree of tourism, urbanization and ecological environment showed a steady increase in Heilongiiang Province from 2003 to 2017, using a coupling coordination degree model. Xiong et al. (2020) examined the spatial-temporal pattern and influencing factor of tourism urbanization of 17 counties in the Dongting Lake region from 2000 to 2018. The study showed that the spatial pattern of coordination degree and coupling degree are low in the middle and high around. Raza et al. (2021) explored the nonlinear relationship among tourism development, economic growth, urbanization and environmental degradation, and analyzes the threshold level of the contribution of tourism development to environmental degradation of top tourism destinations. These research focused on quantifying and evaluating the coupling coordination degree of tourism urbanization and ecology, which ignores the spatial matching relationship between tourism urbanization and resources and environment carrying capacity of different regions.

It is worth noting that the development of tourism urbanization is the integrated part of development in the specific region. Thus, the sustainable development of tourism urbanization can be realized only by clarifying the spatial difference and the spatial coordination relationship between tourism urbanization level and resources and environment carrying capacity and achieving the reasonable allocation of resources on time and space scales. Partitioning, a method considering environmental protection and development potential, is an effective way to provide regional management strategies to maintain ecological sustainability (Wu et al. 2018). At present, this method has been widely applied to study the relationship between socio-economic activities and ecological environment (Hu et al, 2018; Wu et al, 2018; Sun et al, 2020). It can also clearly show the combination state of tourism urbanization level and resources and environment carrying capacity in light of their independent evaluation value. However, few studies have applied this method to the analysis of the spatial matching relationship between tourism urbanization level and resources and environment carrying capacity.

This paper selects the Yellow River Basin as the typical case study. A series of advanced quantitative models such as GIS technology, spatial autocorrelation model and partition method have been used to explore and model the spatio-temporal pattern and matching relationship between tourism urbanization level and resources and environment carrying capacity. Our research focuses on the literature gap on the spatial matching relationship between tourism urbanization level and resources and environment carrying capacity. Specifically, partition method, different from the previous model, is able to consider the coordinated relationship between tourism urbanization level and resources and environment carrying capacity from other dimensions. Further, it identifies the key eco-livable tourism cities on a large scale. This study not only contributes to discovering the time and space heterogeneity of tourism urbanization level and resources and environment carrying capacity, but also fills in the gap on the analysis of the spatial matching relationship between tourism urbanization level and resources and environment carrying capacity. Moreover, our findings can provide the new theoretical reference 
and empirical evidence for the sustainable development of tourism urbanization in the Yellow River Basin.

The remainder of this paper is arranged as follows: the methodology and data section describes the methods and data. The results and discussions section reports and discusses the empirical results. The optimization strategies section proposes sustainable development strategies of tourism urbanization in the Yellow River Basin. Finally, conclusions section summarized the conclusions of this paper.

\section{Methodology and data}

122

\section{Measurement of the tourism urbanization level}

Driven by tourism activities, tourism urbanization contributes to the accumulation and diffusion of productivity factors such as population, capital, and material to tourism-based areas, promoting the orderly expansion of city scale and the continuous improvement of city quality. The quantitative standards on the level of tourism urbanization remain to be clarified in the literature. Based on the recent empirical findings in the development of tourism urbanization (Wang et al. 2017b; Liu et al. 2019), the two most widely used indicators for comprehensive characteristics (tourism industry structure and population structure) are selected in this study, and the ratio of these two indicators is adopted to characterize the contribution of tourism in the development of urbanization. It is calculated as follows:

$$
R=\frac{f}{F} / \frac{m}{M}
$$

where $R$ indicates the tourism urbanization level; $f$ is the income of tourism; $F$ is the total value of industry; $m$ is the number of non-agricultural population; $M$ is the total population.The ratio of tourism income to the total value of the industry reflects the contribution rate of tourism to the national economy. The urbanization rate is a comprehensive manifestation of the urban-rural composition of the population and the development of urban space. The model uses the ratio of these two measures to indicate the strength of the regional tourism industry's impact on the evolution of urbanization. The larger the $R$ value is, the stronger the effect of tourism development on urbanization is, that is, the higher the tourism urbanization level is. At this time, the acceleration of tourism would bring about the large-scale and intensification of urban productivity factors, promote the upgrading of urban industrial structure and the adjustment of employment structure, and thus enhance the promotion of the high-quality development of urbanization. Conversely, the weaker the effect is, the lower the tourism urbanization level is. At this time, it is difficult for the tourism industry to fully exert its effect on the agglomeration of urban factors. As a result, its importance in upgrading the urban industrial structure and adjusting the employment structure is reduced, and its stimulating effect on the demand for non-agricultural employment population and the transfer of rural surplus labor is weakened. 
The carrying capacity of urban resources and environment refers to the economic condition and social activities that can be supported by resources and environment in the existing conditions. (Wang et al. 2017a; Wu et al. 2020)Resources and environmental carrying capacity is closely related to the strategic development of villages, cities, and the country, and the openness and pluralism of the society have promoted its transition from ecology to sociology. It is generally believed that the stability of the composite ecosystem is based on the balance between resources, environment, society, and economy, with the diverse subsystems independent and interrelated (Xu et al. 2003; Feng et al. 2018). Among them, the resource system provides various resources necessary for human survival and development, and resource carrying capacity is the foundation of environmental carrying capacity. The carrying capacity of the environmental system for waste is limited, constituting a constraint on the carrying capacity of resources and the environment. Besides, the resource and environment subsystem

161 is also under pressure from social and economic activities, though it supports and restricts the development of the socio-economic subsystem.

This study is conducted based on recent research findings (Fu et al. 2016; Zhang et al. 2019; Liao et al. 2020). We follow the urban complex ecosystem theory and sustainable development theory, which are combined with the above mentioned conceptual theoretical threshold. Moreover, considering the socio-economic development characteristics of the Yellow River Basin and the internal and external structures of the ecosystem, an evaluation index system of the resources and environment carrying capacity of the Yellow River Basin is constructed. The specific indicators are shown in table 1.

Table 1 Evaluation index system for resources and environment carrying capacity

\begin{tabular}{|c|c|c|c|}
\hline System layer & Index layer (Unit) & Attribute & Weight \\
\hline \multirow[t]{8}{*}{ Economic subsystem } & GDP per capita $(10,000$ yuan $)$ & + & 0.0546 \\
\hline & Proportion of secondary industry in GDP (\%) & + & 0.0137 \\
\hline & Proportion of tertiary industry in GDP (\%) & + & 0.0266 \\
\hline & Total retail sales of consumer goods $(10,000$ yuan $)$ & + & 0.0755 \\
\hline & Local government general budget expenditure (10,000 yuan) & + & 0.0523 \\
\hline & Per capita disposable income of urban residents (yuan) & + & 0.0645 \\
\hline & Per capita annual net income of rural households (yuan) & + & 0.0620 \\
\hline & Investment in fixed assets (10,000 yuan) & + & 0.0737 \\
\hline \multirow[t]{6}{*}{ Social subsystem } & Population density (person/ $/ \mathrm{km}^{2}$ ) & - & 0.0161 \\
\hline & Natural population growth rate $(\%)$ & - & 0.0111 \\
\hline & Per capita area of paved roads in city $\left(\mathrm{m}^{2}\right)$ & + & 0.0431 \\
\hline & Public library collections per million people (volume) & + & 0.0307 \\
\hline & $\begin{array}{l}\text { Number of public transportation vehicles per million } \\
\text { population (unit) }\end{array}$ & + & 0.0656 \\
\hline & Number of beds in health institutions per million people (bed) & + & 0.0332 \\
\hline
\end{tabular}




\begin{tabular}{|c|c|c|c|}
\hline & $\begin{array}{l}\text { Number of students in Colleges and Universities per } 10000 \\
\text { people (person) }\end{array}$ & + & 0.0872 \\
\hline \multirow[t]{4}{*}{ Resource subsystem } & Per capita cultivated land $\left(\mathrm{hm}^{2}\right)$ & + & 0.0388 \\
\hline & $\begin{array}{l}\text { Per capita daily consumption of tap water for residential use } \\
\text { (liter) }\end{array}$ & + & 0.0264 \\
\hline & Volume of water supply (10,000 tons) & + & 0.0500 \\
\hline & Area of land used for urban construction $\left(\mathrm{km}^{2}\right)$ & + & 0.0318 \\
\hline \multirow[t]{8}{*}{ Environmental subsystem } & Per capita green area $\left(\mathrm{m}^{2}\right)$ & + & 0.0459 \\
\hline & Green coverage rate of established areas (\%) & + & 0.0120 \\
\hline & Volume of industrial waste water discharged ( 10,000 tons $)$ & - & 0.0074 \\
\hline & Volume of sulphur dioxide emission (10,000 tons) & - & 0.0115 \\
\hline & Volume of industrial soot(dust) emission (10,000 tons) & - & 0.0016 \\
\hline & Ratio of waste water centralized treated (\%) & + & 0.0195 \\
\hline & Ratio of consumption wastes treated (\%) & + & 0.0168 \\
\hline & Ratio of industrial solid wastes comprehensively utilized (\%) & + & 0.0283 \\
\hline
\end{tabular}

170

171

172

173

174

175

176

177

178

179

180

181

182

183

184

185

186

187

(2) TOPSIS model based on entropy weight

The entropy method determines the weight according to the change degree of the original index data. As an objective weighting method, it avoids the shortcomings of the index weight tending to the subjective consciousness of the evaluators from the weighting methods such as the analytic hierarchy process (AHP) and the Delphi method (Zhao et al. 2020a; Yang et al. 2020). The TOPSIS (Technique for Order Preference by Similarity to an Ideal Solution) method was first proposed by Hwang and Yoon (1981). It is a common method to effectively solve multi-objective decision-making problems in an ideal way (Chen 2019). By combining the entropy weight method with TOPSIS method, this paper overcomes the subjective shortcoming of TOPSIS and can objectively and comprehensively reflect the dynamic changes of the resources and environment carrying capacity of the Yellow River Basin. The specific calculation procedure is as follows ( $\mathrm{Li} 2021)$ :

a) Suppose that there are $m$ objects under study and $n$ evaluation indicators for each object under study. Then, the following judgment matrix can be constructed as follow.

$$
x\left(x_{i j}\right)_{m \times n}(i=1,2, \Lambda, m ; j=1,2 \Lambda, n)
$$

b) The range transformation method is used to standardize the data.

c) Compute information entropy:

$$
H_{j}=-k \sum_{i=1}^{m} p_{i j} \ln p_{i j}, \quad p_{i j}=\frac{x_{i j}}{\sum_{i=1}^{m} x_{i j}}, \quad k=\frac{1}{\ln m}
$$

where $m=79$ 
d) Calculate the weight of index $j: \quad w_{j}=\frac{1-H_{j}}{\sum_{j=1}^{n}\left(1-H_{j}\right)}$

189

where $w_{j} \in[0,1]$.

e) Determine the weighting matrix:

$$
R=\left(l_{i j}\right)_{m \times n}, r=w_{j} \cdot x_{i j}(i=1,2, \Lambda, m, j=1,2, \Lambda, n)
$$

f) Determine the optimal solution $s_{j}^{+}$and the worst solution $s_{j}^{-}$:

$$
s_{j}^{+}=\max \left(r_{1 j}, r_{2 j}, \Lambda, r_{n j}\right), s_{j}^{-}=\min \left(r_{1 j}, r_{2 j}, \Lambda, r_{n j}\right)
$$

g) Calculate the Euclidean distance from each scheme to the positive ideal solution and the negative ideal solution:

$$
s_{i}^{+}=\sqrt{\sum_{j=1}^{n}\left(s_{j}^{+}-r_{i j}\right)^{2}}, s_{j}^{-}=\sqrt{\sum_{j=1}^{n}\left(s_{j}^{-}-r_{i j}\right)^{2}}
$$

h) Calculate the comprehensive evaluation index $c_{i}$ :

$$
C_{i}=\frac{s_{i}^{-}}{s_{i}^{+}+s_{i}^{-}} \quad 0 \leq C_{i} \leq 1
$$

\section{Spatial autocorrelation analysis}

The spatial autocorrelation analysis method is further employed to examine the spatial agglomeration patterns of tourism urbanization level and resources and environment carrying capacity in the Yellow River Basin. Spatial autocorrelation refers to the potential interdependence of some variables in the same distribution area and can effectively measure the spatial agglomeration degree $(\mathrm{Li}$ et al. 2021). There are two main measures in the spatial autocorrelation analysis, namely global and local measures. Global spatial autocorrelation is used to detect the distribution characteristics in the whole region, and it can be calculated as follows (Cheng et al. 2019):

$$
I=\frac{n \sum_{i=1}^{n} \sum_{j=1}^{n} W_{i j}\left(x_{i}-\bar{x}\right)\left(x_{j}-\bar{x}\right)}{\sum_{i=1}^{n} \sum_{j=1}^{n} W_{i j} \sum_{i=1}^{n}\left(x_{i}-\bar{x}\right)^{2}}
$$

where $I$ indicates the global Moran's $I$ index; $n$ is the number of regions; $x_{i}$ and $x_{j}$ are the values of the observed variables at sites of $i$ and $j$ space; $\bar{x}$ is the mean value of $x_{i}$; and $W_{i j}$ represents the spatial weight matrix. The value of Moran's I generally vary between -1 and 1 . Moran's I value greater than 0 indicates positive spatial correlation, while Moran's I value less than 0 indicates negative spatial correlation.

Local spatial autocorrelation can identify whether an attribute value of each space has local 
spatial correlation. It is calculated as follows (Wang et al. 2019):

$$
I_{i}=\frac{x_{i}-\bar{x}}{S_{i}^{2}} \sum_{j=1, j \neq i}^{n} w_{i j}\left(x_{j}-\bar{x}\right)
$$

where $I_{i}$ indicates local Moran's $I$ index; $n$ is the number of regions; $x_{i}$ and $x_{j}$ are the index values of the $i$ and $j$ regions, respectively; $\bar{x}$ is the mean value of $x_{i}$; and $W_{i j}$ represents the spatial weight matrix.

\section{Data source}

In this study, 79 municipal units in the Yellow River Basin are selected as the study area (Zhao et al. 2020b), and the years 2005, 2011 and 2018 are selected as the research time period. The data were collected from the China urban Statistical Yearbook (2006, 2012, 2019), the China Urban Construction Statistical Yearbook (2006, 2012, 2019), the Provincial Statistical Yearbook (2006, 2012, 2019), and the Statistical Bulletin on National Economy and Social Development (SBNESD) of cities in the Yellow River Basin (2005, 2011, 2018). Linear interpolation method was used to pre-process the missing data in the dataset.

\section{Results and discussion}

\section{Spatial distribution pattern}

According to formula (1)-(8), the evaluation index of tourism urbanization and resource environment carrying capacity of the Yellow River Basin in 2005, 2011, and 2018 was calculated. The index values were divided into five levels using the natural discontinuity method, and the spatial pattern distribution maps were reported in Fig. 1 and Fig. 2.

The average values of tourism urbanization in the Yellow River Basin in 2005, 2011, and 2018 were $0.147,0.205$ and 0.384 , respectively, showing a clear upward trend. This finding quantitatively verified that the development of tourism has gradually increased the intensity of its impact on urbanization. As Fig. 1 shows, the agglomeration phenomenon in the spatial distribution of tourism urbanization level was prominent. In 2005, the first and second level cities of tourism urbanization concentrated in Shaanxi Province, the northwestern part of Henan Province, the southern part of Shandong Province, and some cities in Shanxi Province; the fifth-level regions concentrated in Inner Mongolia, Ningxia, and the southeast of Gansu Province. In 2011, the first and second level areas were scattered in Shaanxi Province, Xinzhou City in Shanxi Province, Luoyang City and Kaifeng City in Henan Province, and Tai'an City in Shandong Province; the fifth-level regions were widely distributed in Inner Mongolia and Ningxia, and a small part was scattered in the northern part of the Hexi Corridor and Shandong Peninsula. As of 2018, the first and second level cities concentrated in Shaanxi, Shanxi, and Gansu provinces; the fifth-level regions still concentrated in Inner Mongolia, Ningxia, and the northern part of Shandong Province. Overall, it shows that due to the limitations of economy, resources 

the driving effect of tourism on urbanization is relatively weak, which is consistent with the empirical findings in the existing literature (Ruan et al. 2019).
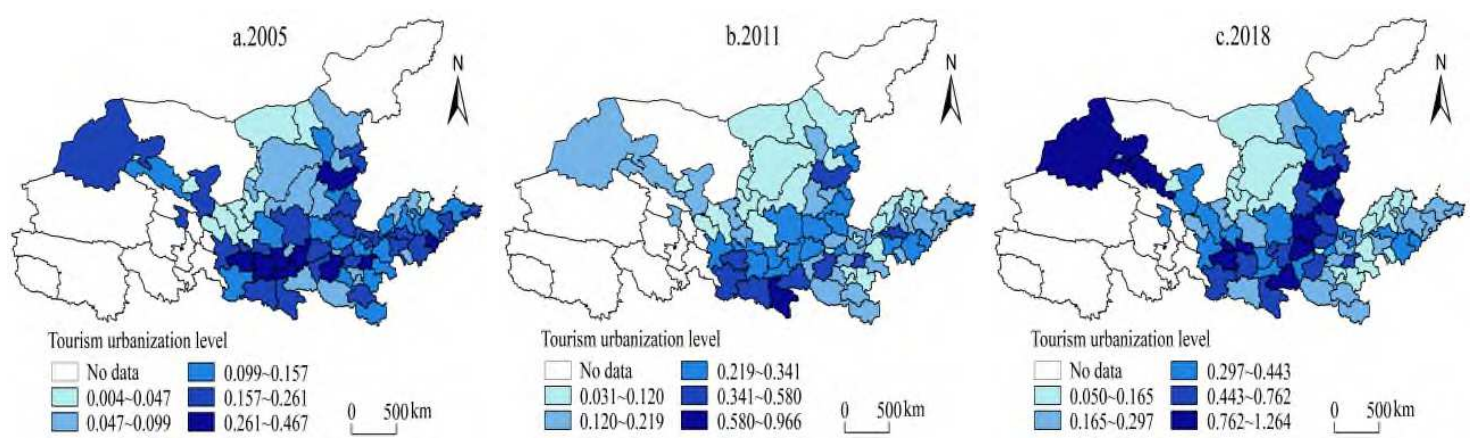

Fig.1 Spatial patterns of tourism urbanization level in the Yellow River Basin

The average resources and environmental carrying capacity of the Yellow River Basin increased from 0.258 in 2005 to 0.425 in 2018 , demonstrating that the resources and environmental carrying capacity of the Yellow River Basin has been significantly improved during the past 14 years. From Fig. 2 , it can be seen that the spatial distribution of resources and environmental carrying capacity exhibited significant "concentration" characteristics. During the study period, the first and second level areas of resources and environmental carrying capacity concentrated in Gansu, Inner Mongolia, and Shandong provinces, and are scattered in the capital cities of Xi'an in Shaanxi Province, Taiyuan in Shanxi Province, and Zhengzhou in Henan Province. The fifth-level cities concentrated in Shaanxi Province, Shanxi Province, and the southeast of Gansu Province, with expanded spatial clusters.
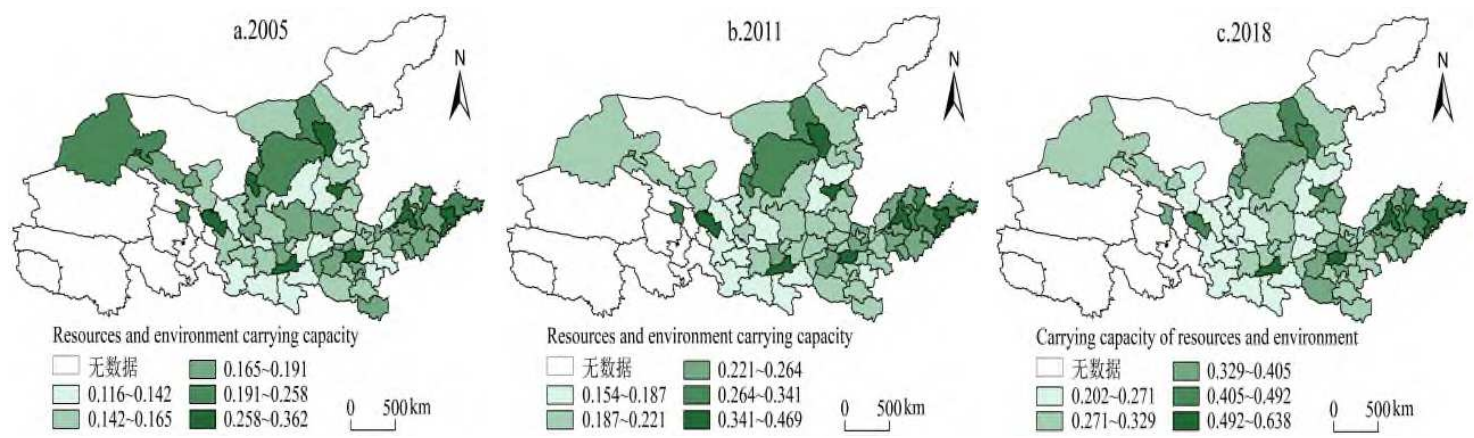

Fig.2 Spatial patterns of resources and environment carrying capacity in the Yellow River Basin

\section{Spatial autocorrelation analysis}

The previous analysis indicated that the types of tourism urbanization level and resources and environmental carrying capacity in the Yellow River Basin had the characteristics of agglomeration distribution in space, implying that there may be spatial correlation in geographical space. To analyze the spatial heterogeneity and dependence of the Yellow River Basin, GeoDa software was used to calculate the global Moran's $I$ index and the local Moran 's $I$ index according to formula (9)-(10). Besides, the LISA agglomeration maps in 2005, 2011 and 2018 were drawn using ArcGIS 10.2 software (Fig. 3 and 4). 
positive and passed the $\mathrm{Z}$ test at the significance level of 5\%, as shown in Table 1. Moran's $I$ index in 2005, 2011 and 2018 were $0.104,0.149$ and 0.380 , respectively. It demonstrates that the level of tourism urbanization in the Yellow River Basin had a positive spatial autocorrelation, and the degree of agglomeration had increased. As illustrated in Fig. 3, the characteristics of local spatial agglomeration of tourism urbanization in the Yellow River Basin were significant. The proportion of positively correlated cities was higher than that of negatively correlated cities, presenting a positive spatial correlation as a whole. Specifically, empirical results include:

(1) The number of cities in high-high agglomeration areas increased from 5 to 13. The scope of this type of area gradually expanded with a significant increase, and the spatial distribution range gradually shifted from the southern part of Shaanxi Province to Shanxi Province. It can be seen that in recent years, Shanxi Province regards tourism as a sustainable alternative industry, and the policy of building a strong country of tourism economy has achieved initial results. The promoting effect of tourism on urbanization in this province is gradually highlighted.

(2) The low-low agglomeration areas were mainly distributed in Gansu and Ningxia provinces in 2005, and gradually concentrated in Ningxia and Shandong provinces in 2018, increasing from 8 to 24 cities. The degree of spatial agglomeration in cities with low levels of tourism urbanization has increased.

(3) The number of cities in high-low agglomeration areas accounted for the lowest proportion. The cities located in this area in 2005 and 2018 were Wuwei and Kaifeng, respectively. The low-high agglomeration areas were relatively unstable in terms of spatial changes.

(4) In 2005, there were 3 low-high agglomeration areas, namely Longnan, Tongchuan and Shangluo. In 2011, the low-high agglomeration area was only Tongchuan City. In 2018, Jiayuguan, Hanzhong, and Taiyuan were mainly the low-high agglomeration areas.

On the whole, the spatially positively correlated areas significantly increased, and the proportion of the number of cities increased from $16.46 \%$ in 2005 to $46.84 \%$ in 2018 , presenting a spatial distribution of clusters. The negative spatial correlation area accounted for a relatively low proportion. In 2005, 2011, and 2018, the proportion was 5.06\%, 1.27\%, and 5.06\%, respectively, exhibiting a discrete distribution in space.

Table 2 Global Moran's I of tourism urbanization level in the Yellow River Basin

\begin{tabular}{cccc}
\hline Year & 2005 & 2011 & 2018 \\
\hline Moran's $I$ & 0.104 & 0.149 & 0.380 \\
$Z(\mathrm{I})$ & 2.181 & 3.170 & 7.329 \\
$P(\mathrm{I})$ & 0.029 & 0.002 & 0.000 \\
\hline
\end{tabular}




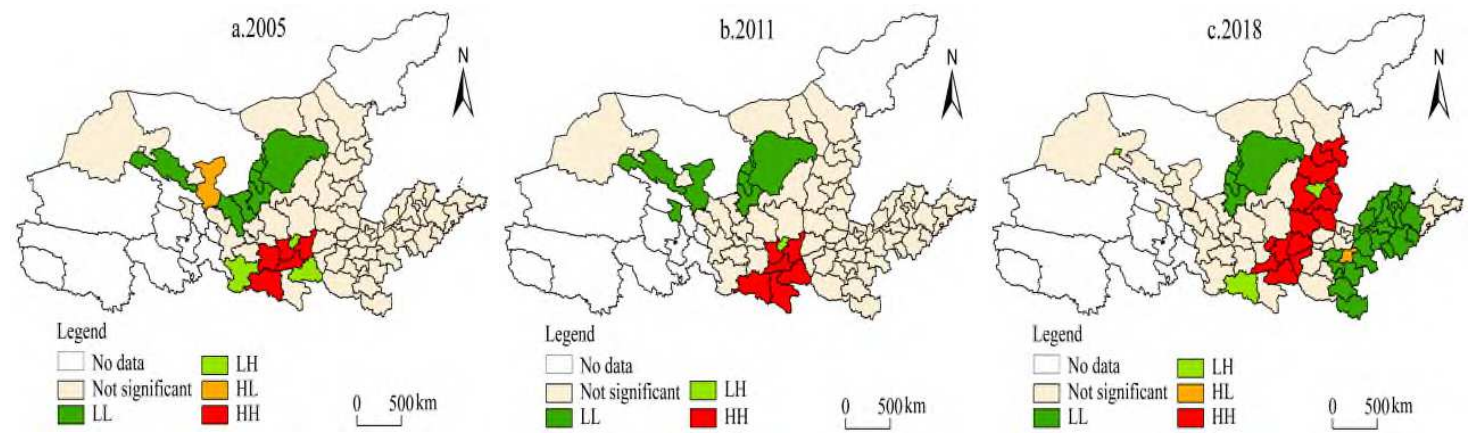

Fig.3 LISA cluster map of tourism urbanization level in the Yellow River Basin

The Global Moran's I index of the resources and environment carrying capacity of the Yellow River Basin was positive. In 2005, Moran's I was close to 0 and failed the significance level test, indicating that the resource and environmental carrying capacity had low spatial autocorrelation and tended to be randomly distributed as a whole. In 2011 and 2018, Moran's I was 0.102 and 0.161, respectively; the $\mathrm{Z}$ values were 1.895 and 2.887 , respectively, both greater than 1.96 , passing the $\mathrm{Z}$ test at the significance level of $5 \%$. Therefore, the carrying capacity of resources and environment had a significant spatial positive correlation in spatial distribution, and the positive correlation gradually increased. As illustrated in Fig. 4, the characteristics of local spatial agglomeration of the resources and environment carrying capacity of the Yellow River Basin were significant. The agglomeration effect mainly occurred in high-high agglomeration areas and low-low agglomeration areas, and the overall spatial correlation was positive. Specifically, empirical results include:

(1) The high-high agglomeration areas in 2005 were mainly Dongying, Zibo, Weifang, and Yantai. In 2011, Binzhou, Linyi, Qingdao, and Weihai were transformed into high-high agglomeration areas. In 2018, Binzhou was withdrawn from the high-high agglomeration type; the types of other cities remained unchanged, and the number of cities increased from 4 to 7. Consequently, the resources and environment carrying capacity of the Yellow River Basin were enhanced in the high-high agglomeration areas, which were mainly concentrated in Shandong Province.

(2) The low-low agglomeration areas in 2005 mainly consisted of four cities, Tianshui, Yan'an, Anyang, and Xinxiang. In 2011, the low-low agglomeration areas included 8 cities: Dingxi, Longnan, Tianshui, Pingliang, Qingyang, Guyuan, Yan'an, and Yuncheng. In 2018, Yuncheng was withdrawn from the low-low agglomeration areas, and the types of other cities remained unchanged. In terms of spatial change, the low-low agglomeration areas gradually evolved from scattered distribution to agglomeration distribution, mainly concentrated in Gansu and Shaanxi provinces.

(3) The high-low agglomeration areas were mainly located in Xi'an, Taiyuan, and Zhengzhou in 2005 and 2011. In 2018, Lanzhou changed to a high-low agglomeration area while Zhengzhou was withdrawn from the high-low agglomeration area, and the types of other cities remained unchanged. The number of cities in high-low agglomeration areas was relatively low with a scattered distribution, mostly in provincial capital cities.

(4) The low-high agglomeration areas in 2005 were mainly Ulan Qab and Linyi, changed to Ulan 
Qab, Dezhou, and Rizhao in 2011 and Dezhou, Binzhou, and Rizhao in 2018.

On the whole, the positive spatial correlation area increased, with the proportion of the number of cities increasing from $10.13 \%$ in 2005 to $17.72 \%$ in 2018 , presenting an agglomeration distribution in space. There were few negatively correlated cities in space, with the proportion of cities increasing from $6.33 \%$ in 2005 to $7.59 \%$ in 2018 , exhibiting a relatively fragmented distribution in space.

Table 3 Global Moran's I of resources and environment carrying capacity in the Yellow River Basin

\begin{tabular}{cccc}
\hline Year & 2005 & 2011 & 2018 \\
\hline Moran's $I$ & 0.010 & 0.102 & 0.161 \\
$Z(\mathrm{I})$ & 0.430 & 1.895 & 2.887 \\
$P(\mathrm{I})$ & 0.290 & 0.034 & 0.003 \\
\hline
\end{tabular}
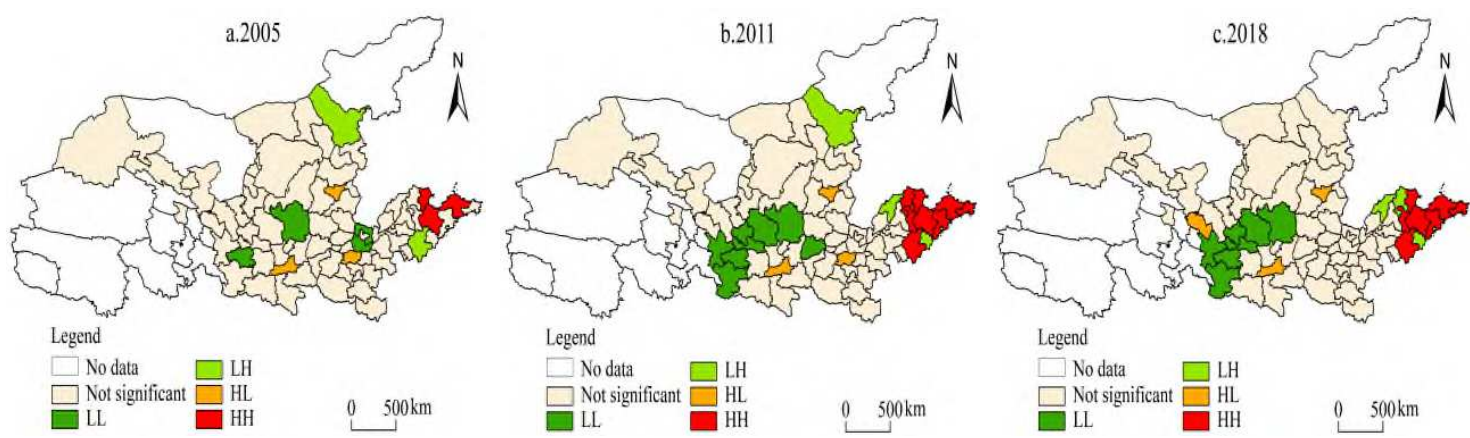

Fig.4 LISA cluster map of resources and environment carrying capacity in the Yellow River Basin

\section{7}

\section{Space type division}

The above analysis revealed that the tourism urbanization level and resources and environment carrying capacity in the Yellow River Basin were unevenly distributed in space. Therefore, the coordinated spatial characteristics of the two needed to be explored. In this paper, a two-dimensional scatter diagram was employed to classify its spatial combination types, dividing the 79 cities in the Yellow River Basin into 5 types (Fig. 7). It included high level of tourism urbanization-high resources and environmental carrying capacity regions $(\mathrm{H}-\mathrm{H})$, high level of tourism urbanization-low resources and environmental carrying capacity regions $(\mathrm{H}-\mathrm{L})$, low level of tourism urbanization — high resources and environmental carrying capacity regions (L-H), low level of tourism urbanization-low resources and environmental carrying capacity regions (L-L), and medium-sized tourism urbanization-medium resources and environmental carrying capacity regions (M-M), corresponding to 5 areas of I, II, III, IV, and $\mathrm{V}$ in Fig. 7. Furthermore, with the help of ArcGIS10.2 software, the spatial pattern of the combination types of tourism urbanization level and resources and environment carrying capacity in 2005, 2011 and 2018 was drawn (Fig. 8). The analysis can be described as follows:

(1) In 2005, there were $6 \mathrm{H}-\mathrm{H}$ cities, accounting for $7.59 \%$ of the total. Most of them were regions with prominent economic advantages, including Qingdao, Weihai, Jiuquan, Zhengzhou, Xi'an, and Xining. These cities had a high degree of coordination between the level of tourism urbanization and the carrying capacity of resources and environment. In 2011 and 2018, the 
number of $\mathrm{H}-\mathrm{H}$ cities dropped to zero, showing a vacant state. Thus, the carrying capacity of resources and the environment had a greater restrictive effect on the current high-level areas of tourism urbanization, and the spatial correspondence between the two was weak.

(2) In 2005, there were $16 \mathrm{H}-\mathrm{L}$ cities, accounting for $20.25 \%$ of the total, scattered in various provinces, such as Datong, Tianshui, Kaifeng, and Weinan. By 2018, the number of H-L cities increased to 19 , accounting for $24.05 \%$ of the total, concentrated in Shanxi Province, the northwestern part of Gansu Province, and the southern part of Shaanxi Province. Notably, the proportion of H-L cities in Shanxi Province increased from $45.45 \%$ in 2005 to $72.73 \%$ in 2018, demonstrating that the spatial coordination between the tourism urbanization level and resources and environment carrying capacity in most cities in Shanxi Province was relatively low and needed to be optimized urgently.

(3) M-M cities were reduced from 38 in 2005 to 30 in 2018, accounting for more than one-third of the total. They were the most numerous and widely distributed type. Its spatial pattern changed from the original contiguous distribution to the central concentration. These areas had a moderate level of tourism urbanization or the carrying capacity of resources and environment, and there were certain deficiencies in both the level of tourism urbanization and the carrying capacity of resources and environment, leaving a large room for improvement.

372

(4) The number of L-H cities increased from 7 to 10, with a relatively small proportion. Its spatial pattern showed a trend of continuously gathering in the eastern part of Shandong Province. The urbanization and the carrying capacity of resources and the environment gradually decreased, and the number of regions with low-level coordination (L-L) and unbalanced (H-L or L-H) increased, further 

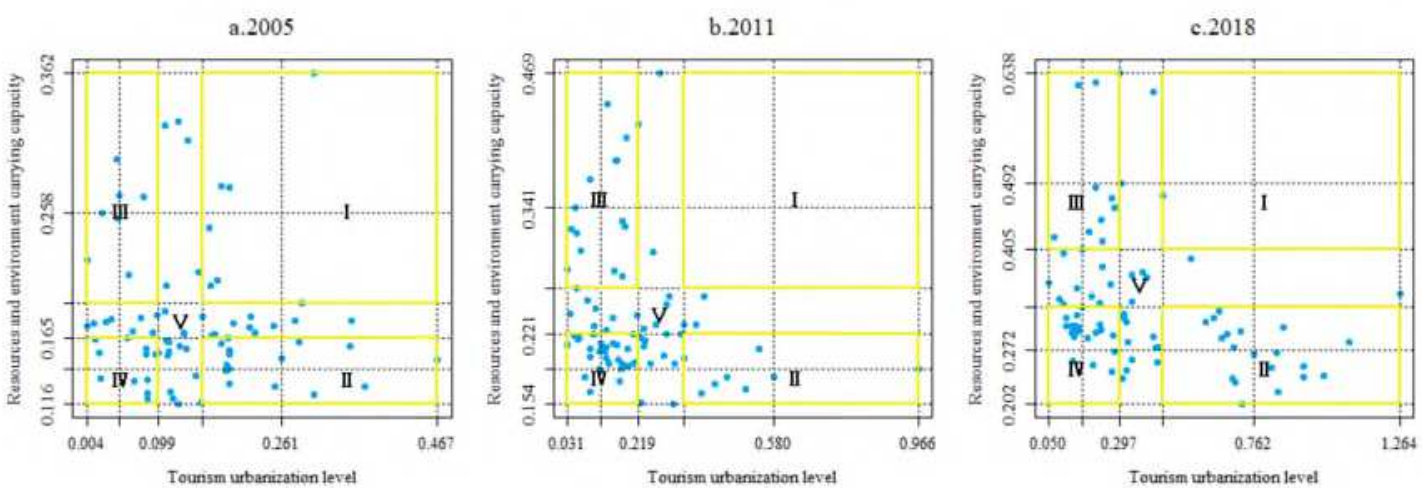

Note: X-Y model can be used to represent the combination type of tourism urbanization level and resources and environment carrying capacity, where $\mathrm{X}$ represents the tourism urbanization level and $\mathrm{Y}$ represents the level of resources and environment carrying capacity. The classification standard is shown in Fig.1 and Fig.4

Fig.7 Combination types of tourism urbanization and resources and environment carrying capacity

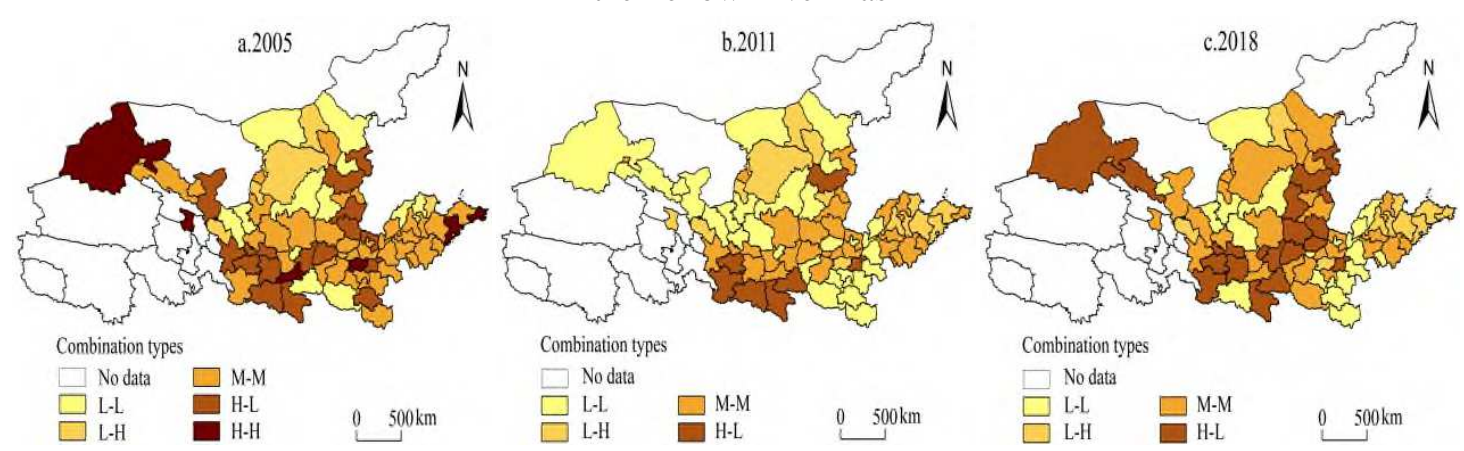

Fig.8 Spatial pattern of combination types of tourism urbanization and resources and environment carrying capacity in the Yellow River Basin

\section{Optimization strategies}

Empirical results in this paper indicate that in order to promote the sustainable development of tourism urbanization in the Yellow River Basin, decision makers and government officials formulate different and targeted regional strategies according to their development characteristics. Therefore, this paper proposes the following policy recommendations.

Firstly, the findings from this research showed that the characteristics of spatial agglomeration of tourism urbanization in the Yellow River Basin are significant. Therefore, cross-regional cooperation in tourism urbanization could be actively promoted (Wang et al. 2017b). High-High agglomeration areas can break the traditional administrative boundary constraints, formulate the direction and goals of the overall tourism urbanization development, and build a unified system and mechanism. On this basis, the free flow and efficient allocation of various elements of tourism urbanization are further promoted, forming a new layout for the development of high-quality tourism urbanization with high-level cluster development and complementary advantages.

Secondly, the spatial differences of resources and environmental carrying capacity in the Yellow River Basin are significant, among which the high-value areas concentrated in Shandong Province. 
Thus, the related departments could fully demonstrate the advanced demonstration role of Shandong Province and other eastern regions, accelerate the optimization of layout and the deconstruction of functions, and promote cities in the central and western regions where the utilization of resources and environmental conditions is not reasonable enough and sufficient to upgrade together. Decision makers could break down the system and mechanism barriers, increase the degree of openness, and attract high-end elements, so as to improve the resources and environment carrying capacity in a more comprehensive and coordinated manner.

Thirdly, for the development of tourism urbanization in the whole river basin, the carrying capacity of resources and environment could be considered as rigid constraints in a forward-looking manner. To take the advantage of the opportunity of China's ecological civilization construction, the ecological civilization could be fully integrated into the entire process of tourism urbanization, and the development and protection of a compatible new tourism urbanization development model need to be explored (Zhang et al. 2020). Simultaneously, from the perspective of the natural environment, it is necessary to steadily strengthen the protective effect of the carrying capacity of resources and environment on the construction of tourism urbanization, realizing the coordinated development of tourism urbanization and the carrying capacity of resources and environment.

Lastly, as the empirical results of space type division show, the resources and environment carrying capacity of the Yellow River Basin has a great restrictive effect on the current high-level areas of tourism urbanization, and the spatial correspondence between the two is weak. Therefore, H-L cities could promote the timely and rapid development of tourism urbanization under the new normal, improve relevant environmental standards, and tighten environmental access systems(Raza et al. 2021). Meanwhile, such cities can optimize the spatial layout of tourism urbanization according to the environmental carrying capacity and environmental quality, and strive to realize the "new normal of tourism urbanization", in order to become a typical demonstration area for the construction of eco-tourism livable cities.

\section{Conclusions}

In this study, with the Yellow River Basin as the research area and 2005, 2011, and 2018 as the research time nodes, GIS technology, spatial autocorrelation model and partition method were adopted to model and analyse the spatio-temporal pattern and matching relationship between tourism urbanization level and resources and environment carrying capacity. The main findings of this research is as follows:

From the perspective of temporal and spatial patterns, the tourism urbanization level and resources and environment carrying capacity in the Yellow River Basin increased while there were significant regional differences. In terms of tourism urbanization evaluation, Shaanxi Province maintains the leading position, while Shanxi Province has great development potential. However, the characteristics of tourism urbanization in Inner Mongolia and Ningxia are not significant. As for the evaluation of resources and environment carrying capacity, the high value areas concentrated in Gansu, Inner Mongolia and Shandong provinces, while the low value areas concentrated in Shaanxi, Shanxi and the 
southeast of Gansu Province.

From the perspective of spatial autocorrelation, the level of tourism urbanization in the Yellow River Basin always had a significant positive spatial correlation, and the degree of agglomeration increased. However, the carrying capacity of resources and environment had a significant positive spatial correlation in 2011 and 2018. It failed to pass the significance level test in 2005, and was not statistically significant. Overall it was random distributed. The local autocorrelation LISA agglomeration map indicated that the local spatial autocorrelation between the tourism urbanization level and resources and environment carrying capacity was significant. Among them, the number of spatially positively correlated cities was more than that of negatively correlated cities, and the cities were spatially positively correlated on the whole. The positive correlation area and the negative correlation area showed a distinct distribution difference; the former was mostly concentrated, while the latter was mostly scattered.

The coordination relationship between the tourism urbanization level and resources and environment carrying capacity in the Yellow River Basin was continuously adjusted. Areas with a positive interaction $(\mathrm{H}-\mathrm{H})$ between the level of tourism urbanization and the carrying capacity of resources and the environment were distributed in dots. Its number was small and dropped to zero. The number of regions with low-level coordination (L-L) and imbalances (H-L or L-H) increased. The carrying capacity of resources and the environment exhibited a greater restrictive effect on the current high-level areas of tourism urbanization, and the spatial correspondence between the two was weak.

The results of this study have made substantial contributions to the development of comprehensive partitions and optimization strategies to achieve sustainable tourism urbanization in the Yellow River Basin, future research can be conducted in the following directions. First, the definition of the connotation of the tourism urbanization and resources and environment carrying capacity and the construction of the index system remain unclear. How to set a more standardized and effective evaluation mechanism based on the operating characteristics of the two systems still needs to be further discussed in follow-up research. Second, tourism urbanization, as a highly environment-dependent urbanization development model, has an inseparable relationship with the carrying capacity of resources and environment. How to describe the interaction effect and evolution process between the two from the micro level? How to define a reasonable scale of tourism urbanization development based on the threshold of resources and environmental carrying capacity? These complications merits further investigations in future research.

Acknowledgments We sincerely thank the editors and reviewers for their patience and comments.

Authors' contributions This idea was given by WZF and CQQ. WZF was a major contributor in 483 writing the manuscript. CQQ participated in the review and modification of the manuscript. Both authors read and approved the final manuscript. 
Funding This study was supported by National Natural Science Foundation of China (No. 41771162).

Data availability The datasets used in this research are available on reasonable request.

\section{Compliance with ethical standards}

Competing interests The authors declare no competing interests.

Ethics approval and consent to participate Not applicable

Consent for publication Not applicable.

\section{References}

Chen PY (2019) Effects of normalization on the entropy-based TOPSIS method. Expert Systems with Applications, 136. https://doi.org/10.1016/j.eswa.2019.06.035

Cheng X, Long R, Chen H, \& Li QW (2019) Coupling coordination degree and spatial dynamic evolution of a regional green competitiveness system - A case study from China. Ecological Indicators, 104(SEP.): 489-500. https://doi.org/10.1016/j.ecolind.2019.04.003

Feng ZM, Sun T, Yang YZ, \& Yan HM (2018) The Progress of Resources and Environment Carrying Capacity: from Single-factor Carrying Capacity Research to Comprehensive Research. Journal of Resources and Ecology, 9(2).

Fu J, Zang C, Zhang J (2020) Economic and resource and environmental carrying capacity trade-off analysis in the Haihe River basin in China. Journal of Cleaner Production, 270(3):122271. https://doi.org/10.1016/j.jclepro.2020.122271

González SA, Yáñez-Navea K, \& Muñoz M (2014) Effect of coastal urbanization on sandy beach coleoptera Phaleria maculata (Kulzer, 1959)in northern Chile. Marine Pollution Bulletin, 83(1): 265-274. https://doi.org/10.1016/j.marpolbul.2014.03.042

Rai B, \& Goswami S (2019) Urbanization and Tourism Induced Challenges in Waste Management in Hill Towns: Case of Gangtok: Proceedings of 6th IconSWM 2016. http://libdb.csu.edu.cn:80/rwt/SPRINGERLINK/https/MSYXTLUQPJUB/10.1007/978-981-10-7290-1 $-34$

Hu MM, Li ZT, Yuan MJ, Fan C, \& Xia BC (2019) Spatial differentiation of ecological security and differentiated management of ecological conservation in the Pearl River Delta, China. Ecol. Indicat. 104, 439e448. https://doi.org/10.1016/j.ecolind.2019.04.081

Hwang CL, \& Yoon KS (1981) Multiple attribute decision making. Spring-verlig, Berlin. Izquierdo AE, Grau HR, Navarro CJ, Casagranda E, Castilla MC, \& Grau A. (2018) Highlands in transition: Urbanization, pastoralism, mining, tourism, and wildlife in the Argentinian Puna. Moun tain Research and Development, 38, 390-400. 10.1659/MRD-JOURNAL-D-17-00075.1 
Li JS, Sun W, Li MY, \& Meng LL (2021) Coupling coordination degree of production, living and ecological spaces and its influencing factors in the yellow river basin. Journal of Cleaner Production, 298, 126803. https://doi.org/10.1016/j.jclepro.2021.126803

525 Li X (2021) TOPSIS model with entropy weight for eco geological environmental carrying capacity assessment. Microprocessors and Microsystems, 103805. https://doi.org/10.1016/j.micpro.2020.103805 Liao SJ, Wu Y, Wong SW, \& Shen LY (2020) Provincial perspective analysis on the coordination between urbanization growth and resource environment carrying capacity (RECC) in China. Science of The Total Environment, 730:138964. https://doi.org/10.1016/j.scitotenv.2020.138964Ge Liu YJ, Tang JX, \& Ma XF (2019) Spatial and temporal evolution and model of tourism urbanization in concentrated continuous poor areas: taking Wuling Mountainous Area in Hunan as an example. Economic Geography, 39(10): 214-222+230 (in Chinese).

533 Nitivattananon V, \& Srinonil S (2019) Enhancing coastal areas governance for sustainable tourism in 534 the context of urbanization and climate change in eastern Thailand. Advances in Climate Change Research, 3, 47-58. https://doi.org/10.1016/j.accre.2019.03.003

536 Raza SA, Qureshi MA, Ahmed M, Qaiser S, Ali R, \& Ahmed F (2021) Non-linear relationship between 537 tourism, economic growth, urbanization, and environmental degradation: evidence from smooth 538 transition models. Environmental Science and Pollution Research, 28:1426-1442. 539 https://doi.org/10.1007/s11356-020-10179-3

540 Ruan WQ, Li YQ, Zhang SN, \& Liu CH (2019) Evaluation and drive mechanism of tourism ecological 541 security based on the DPSIR-DEA model. Tourism Management, 75(609-25). 542 https://doi.org/10.1016/j.tourman.2019.06.021

543 Sun MY, Li XH, Yang RJ, Zhang Y, Zhang L, Song ZW et al (2020) Comprehensive partitions and 544 different strategies based on ecological security and economic development in Guizhou Province, 545 China[J]. Journal of Cleaner Production, 274(1):122794. https://doi.org/10.1016/j.jclepro.2020.122794

546 Tao H, Liu JM, Deng Y, \& Du Ao (2017) Tourism sectorization opportunity spectrum model and space 547 partition of tourism urbanization area: a case of the Mayangxi ecotourism area, Fujian province, China.

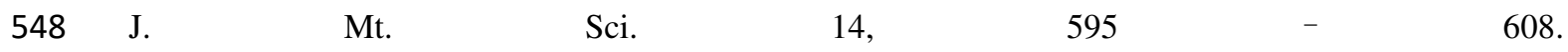

549 http://libdb.csu.edu.cn:80/rwt/SPRINGERLINK/https/MSYXTLUQPJUB/10.1007/s11629-015-3747-8

550 Wei SC (2017) Review and prospects of research on tourism urbanization. Shanghai Land \& 551 Resources.

552 Wang KF, Zhao XK, Peng BY, \& Zeng YM (2020) Spatio-temporal pattern and driving factors of 553 municipal solid waste generation in China: New evidence from exploratory spatial data analysis and 554 dynamic spatial models. Journal of Cleaner Production, 270. 555 https://doi.org/10.1016/j.jclepro.2020.121794

556 Wang R, Cheng JH, Zhu YL, \& Lu PX (2017a) Evaluation on the coupling coordination of resources 557 and environment carrying capacity in Chinese mining economic zones. Resources Policy. 558 https://doi.org/10.1016/j.resourpol.2017.05.012

559 Wang XY, Liu EL, \& Hou JJ (2017b) Characteristics and types of spatial-temporal differentiation of 
tourism urbanization response in Shandong province. Scientia Geographica Sinica, 37(7): 1087-1094 (in Chinese).

Wu MW, Wu JQ, \& Zang CF (2020) A comprehensive evaluation of the eco-carrying capacity and green economy in the Guangdong-Hong Kong-Macao Greater Bay Area, China. Journal of Cleaner Production, 281(1):124945. https://doi.org/10.1016/j.jclepro.2020.124945

Wu X, Liu SL, Cheng FY, Hou XY, Zhang YQ, Dong SK, \& Liu GH (2018) A regional strategy for ecological sustainability: a case study in Southwest China. Sci. Total Environ. 616, 1224e1234. https://doi.org/10.1016/j.scitotenv.2017.10.196.

Xie PF, Chandra V, \& Gu K (2013) Morphological changes of coastal tourism: A case study of Denarau Island, Fiji[J]. Tourism Management $\quad$ Perspectives, $\quad 5(1)$ : 75-83. https://doi.org/10.1016/j.tmp.2012.09.002

Xinhua News Agency, 2019. General secretary Xi Jinping delivered an important speech at the Symposium on ecological protection and high-quality development of the Yellow River Basin. Res. Soil Water Conserv. 1e2 https://doi.org/10.14123/j.cnki.swcc.2019.0226.

Xiong JX, Wang WH, He SH, Yi Y \& Tang CF (2020) Spatio-temporal pattern and influencing factor of coupling coordination of tourism urbanization system in the Dongting Lake Region. Scientia Geographica Sinica, 40(9): 1532-1542. https://doi.org/10.13249/j.cnki.sgs.2020.09.015

Xu LY, Yang ZF, \& Li W (2003) Review on urban ecosystem carrying capacity. Urban Environment \& Urban Ecology, (6), 60e62.

Yang T, Zhang Q, Wan XH, Li XP, Wang YY, \& Wang W (2020) Comprehensive ecological risk assessment for semi-arid basin based on conceptual model of risk response and improved TOPSIS model-a case study of wei river basin, china. The Science of the Total Environment, 719(Jun.1), 137502.1-137502.16. https://doi.org/10.1016/j.scitotenv.2020.137502

Zhang F, Wang Y, Ma XJ, Wang Y, Yang GC \& Zhu L (2019) Evaluation of resources and environmental carrying capacity of 36 large cities in China based on a support-pressure coupling mechanism[J]. The Science of the Total Environment, 688(Oct.20):838-854. https://doi.org/10.1016/j.scitotenv.2019.06.247

Zhang T, \& Li L (2020) Research on temporal and spatial variations in the degree of coupling coordination of tourism-urbanization-ecological environment: a case study of heilongjiang, china. Environment Development and Sustainability(2), 1-18. https://doi.org/10.1007/s10668-020-00976-8

Zhao LL, Li JY, \& Shao, QL (2020a) Evaluation of urban comprehensive carrying capacity: case study of the Beijing-Tianjin-Hebei urban agglomeration, China. Environ Sci Pollut Res 27, 19774-19782.http://libdb.csu.edu.cn:80/rwt/SPRINGERLINK/https/MSYXTLUQPJUB/10.1007/s1135 $\underline{6-020-08463-3}$

Zhao JJ, Liu Y, Zhu YK, Qin SL, Wang YH, \& Miao CH (2020b) Spatio-temporal differentiation and influencing factors of the coupling and coordinated development of new urbanization and ecological environment in the Yellow River Basin. Resources Science, 42(1): 159-171 (in Chinese). 